Published in final edited form as:

Acc Chem Res. 2008 December ; 41(12): 1685-1695. doi:10.1021/ar8000348.

\title{
The Pursuit of a Scalable Nanofabrication Platform for Use in Material and Life Science Applications
}

\author{
STEPHANIE E. A. GRATTON $\dagger, \ddagger, \S$, STUART S. WILLIAMS ${ }^{\dagger} \ddagger$, MARY E. NAPIER ${ }^{\dagger}, \ddagger, \S$, PATRICK \\ D. POHLHAUS ${ }^{\dagger}, \S, \perp$, ZHILIAN ZHOU $\perp$, KENTON B. WILES ${ }^{\dagger}$, BENJAMIN W. MAYNOR ${ }^{\dagger}, \perp$, \\ CLIFTON SHEN\#, TOVE OLAFSEN $§, \#$, EDWARD T. SAMULSKI ${ }^{\dagger} \ddagger$, and JOSEPH M. \\ DESIMONE $\dagger, \ddagger, \S, \perp, \|,{ }^{*}$ \\ $\uparrow$ Department of Chemistry, University of North Carolina at Chapel Hill, Chapel Hill, North Carolina 27599 \\ \$Institute for Advanced Materials, University of North Carolina at Chapel Hill, Chapel Hill, North Carolina \\ 27599 \\ $\S$ Carolina Center of Cancer Nanotechnology Excellence, University of North Carolina at Chapel Hill, Chapel \\ Hill, North Carolina 27599
}

$\perp$ Liquidia Technologies, P.O. Box 110085, Research Triangle Park, North Carolina 27709

|| Department of Chemical and Biomolecular Engineering, North Carolina State University, Raleigh, North Carolina 27695

\# Department of Molecular and Medical Pharmacology, University of California at Los Angeles, Los Angeles, California 90095

\section{CONSPECTUS}

In this Account, we describe the use of perfluoropolyether (PFPE)-based materials that are able to accurately mold and replicate micro- and nanosized features using traditional techniques such as embossing as well as new techniques that we developed to exploit the exceptional surface characteristics of fluorinated substrates. Because of the unique partial wetting and nonwetting characteristics of PFPEs, we were able to go beyond the usual molding and imprint lithography approaches and have created a technique called PRINT (Particle [or Pattern] Replication In Nonwetting Templates).

*Corresponding author. E-mail: desimone @unc.edu. 


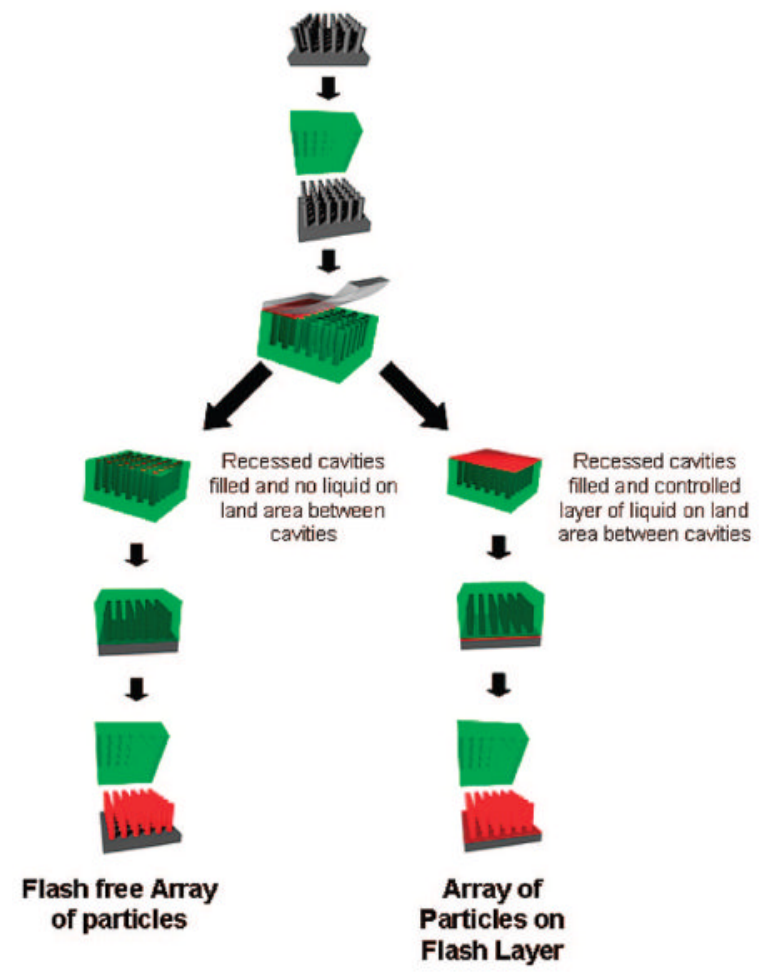

PRINT is a distinctive "top-down" fabrication technique capable of generating isolated particles, arrays of particles, and arrays of patterned features for a plethora of applications in both nanomedicine and materials science. A particular strength of the PRINT technology is the high-resolution molding of well-defined particles with precise control over size, shape, deformability, and surface chemistry. The level of replication obtained showcases some of the unique characteristics of PFPE molding materials. In particular, these materials arise from very low surface energy precursors with positive spreading coefficients, can be photocured at ambient temperature, and are minimally adhesive, nonswelling, and conformable. These distinctive features enable the molding of materials with unique attributes and nanometer resolution that have unprecedented scientific and technological value. For example, in nanomedicine, the use of PFPE materials with the PRINT technique allows us to design particles in which we can tailor key therapeutic parameters such as bioavailability, biodistribution, target-specific cell penetration, and controlled cargo release. Similarly, in materials science, we can fabricate optical films and lens arrays, replicate complex, naturally occurring objects such as adenovirus particles, and create 2D patterned arrays of inorganic oxides.

\section{Introduction}

Replication of submicrometer features is a challenging materials problem. The past few decades have witnessed the emergence of soft lithography as an important tool for low cost pattern replication on the micrometer and nanometer scale. ${ }^{1}$ Soft lithography uses embossing and stamping techniques with applied forces as an alternative to photolithography for the manufacture of integrated circuits and other devices with sub-50 $\mathrm{nm}$ feature sizes. Embossing typically involves the patterning of materials such as polymers, organics, and biological molecules into continuous arrays of patterned features using molds made from either hard materials (quartz/glass, glassy polymers) or soft elastomeric materials to generate features that form on top of an interconnecting flash layer. ${ }^{1-3}$ The field of soft lithography has traditionally been dominated by the elastomer poly(dimethylsiloxane) (PDMS). ${ }^{4,5}$ Despite the advantage of PDMS for use in soft lithography, it has been shown to suffer from serious drawbacks 
including the fact that it swells in common organic solvents and is known to leave cyclic silicone derivatives on surfaces being molded or patterned. ${ }^{6}$ A recent breakthrough by Rolland et al. exploits the excellent solvent resistance and the inherent release properties of highly fluorinated perfluoropolyether (PFPE) elastomers as an exceptional molding material. ${ }^{7,8}$ The unique range of material properties of PFPE-based elastomers-chemical resistance, extremely low surface energy, high gas permeability, solvent resistance, high elastic recovery and good mechanical strength-translates into the ability of PFPEs to mold most organic and aqueous liquids to generate useful materials in the form of isolated particles, arrays of particles, and arrays of patterned features for a number of applications in both nanomedicine and materials science.

Embossing is the process of creating a three-dimensional image or design in paper and in ductile materials. It is typically accomplished with a combination of heat and pressure. Unlike embossing, PFPE-based molding opens up unique approaches that exploit wetting, partial wetting, and nonwetting phenomena instead of relying on heat and pressure associated with traditional embossing approaches. As a result of the very low surface energy and high gas permeability of PFPE-based materials, we are able to mold materials by exploiting the ability to "dead end" fill recessed cavities in PFPE molds with a wide range of organic liquids. Depending on the exact details of how the filling process is completed (Figure 1), including the exact nature of the liquid to be molded, we can uniquely fabricate isolated particles, arrays of particles, and arrays of patterned features using a combination of cavity filling and free meniscus coating concepts. We have reported the fabrication of features ranging in size from $2 \mathrm{~nm}$ to tens of micrometers and have demonstrated that we are able to accurately mold and replicate nanometer-scale features with a resolution of $0.4 \mathrm{~nm} .{ }^{9}$

In addition to the unprecedented resolution enabled by the use of PFPE-based materials in molding processes, we find that we can fill recessed cavities within the PFPE molds with most organic and aqueous liquids without wetting the land area between the cavities (Figure 1). As such, once the liquid contained in the cavities is solidified, discrete objects in the mold can be achieved without the formation of the ubiquitous "flash" or "scum" layer. The flash layer is common to traditional embossing or soft lithography techniques where applied forces are used. 7,8,10,11 With PFPE-based molds, we can fabricate harvestable, flash-free objects or particles using a process called PRINT (Particle [or Pattern] Replication In Nonwetting Templates). The PRINT process begins with the formation of a master template, typically an etched silicon wafer formed using advanced lithographic techniques (Figure 1A), which is coated with a photocurable liquid PFPE that is evenly distributed across the surface of the master template. Once the liquid fluoropolymer has completely wet the master template, it is photochemically cross-linked and subsequently peeled away to generate a precise mold having nanoscale cavities (Figure 1B). For the fabrication of two-dimensional arrays of particles or free particles, the PFPE mold is filled with an appropriate liquid via capillary filling without wetting the land area around the cavities (Figure 1C). The liquid in the mold cavities is then converted to a solid using a wide range of methods including curing chemistries, evaporation, lyophilization, or liquid to solid phase transitions (Figure 1E,E'). The resultant particles can be removed from the mold and transferred to another surface to generate a two-dimensional array or to yield free particles (Figure 1F,F',G).

PRINT is unique from the imprint lithography techniques promulgated by Whitesides et al. ${ }^{1}$, 4,12 in that PRINT uses elastomeric fluoropolymers instead of silicones, which results in three important distinctions: (i) perfluoropolyether elastomers have a lower surface energy, which facilitates the selective filling of nanoscale cavities in the mold with almost any organic liquid without wetting the land area around the cavities, which enables distinct objects or particles to be formed even at the micro- and nanoscale; (ii) organic liquids do not swell fluoropolymers as they do silicones, allowing for the fabrication of a wide range of organic particles with 
desired attributes (surface chemistries, degradation characteristics, deformability) (Figure 2); (iii) the Teflon-like characteristics of the fluoropolymer mold allow the resultant organic particles to be easily harvested or removed from the mold.

Herein we describe two research areas being pursued by our laboratory; nanoparticles for therapeutic and imaging applications and nanopatterned films for materials applications. Preliminary in vitro and in vivo studies using PRINT particles is presented. The use of PRINT technology in materials applications, such as optical film and lens applications, replication of naturally occurring objects, and 2D array patterning of inorganic oxides are also discussed.

\section{Nanomedicine}

Establishing the interdependency of the size, shape, deformability, and surface chemistry of micro- and nanoparticles in vitro and in vivo over length scales ranging from cells to tissues to the entire organism are needed by many different research communities including environmental regulators, pulmonologists, oncologists, pharmaceutical scientists, toxicologists, cell biologists and dermatologists. There is a need for definitive answers related to particle biodistribution maps based on changes in particle size, shape, deformability, and surface chemistry using "calibration quality" particles. These nanofabricated tools (e.g., precisely defined particles) hold significant promise to provide insight into the fundamentals of cellular and biological processes because they can yield essential insights into the design of effective vectors for use in nanomedicine. Beyond understanding the biodistribution of particles delivered via parenteral routes, particle size, shape, deformability, and surface chemistry should play a very significant role in understanding the mechanisms associated with particle inhalation, either intentionally for use as a therapeutic or during environmental exposure.

There is a significant need to establish such definitive bio-distribution maps for nanomaterials. Heretofore essentially all of what is known about the biodistribution, pharmacokinetics, and pharmacodynamics of "particles" in vitro and in vivo has been garnered using either ill-defined particles or systems where there is little control to systematically vary the key interdependent variables of size, shape, deformability, and surface chemistry. Liposomal systems have been the focus of many studies over the last 40 years and have led to the "established" understandings of where nanomaterials partition in vivo, what sizes lead to cellular internalization and tumor accumulation, for example. ${ }^{13,14}$ Unfortunately, liposomal systems can be very misleading probes since they are dynamic, constantly equilibrating, self-assembled entities whose shape and surface chemistry is ill-defined especially when placed into the biological milieu where equilibration reactions occur with naturally occurring lipidic membranes. Beyond liposomes, most all other nanoparticle systems that have been reported have essentially no ability to control the size or shape of particles in a defined way to determine the design rules for biodistribution or cellular internalization and intracellular trafficking. ${ }^{10}$ In addition, there is also no ability with liposomes and many traditional nanoparticle systems to systematically hold particle size and shape constant and iterate on the range of surface characteristics such as ligand type, spatial distribution of ligands, and stoichiometry of the ligands so as to understand the issues of multifunctional or multiplexed particles. On top of these shortcomings, there is certainly no way of using traditional particle or liposomal systems to understand how a deformable particle or object of precisely defined size, shape, and surface chemistry can dynamically circumvent various biological barriers. Understanding the role that mechanobiology plays as a function of size, shape, and surface chemistry certainly lies at the core of how biological "particles" like neutrophils and red blood cells navigate the barriers that confront them. ${ }^{15}$ Ascertaining definitive biodistribution maps through the use of precisely defined particle probes containing appropriate imaging beacons useful for quantification will undoubtedly lead to a set of rules 
that will be of immense use to science and to the application of nanocarriers for improved human health, treatment, and diagnosis.

PRINT enables a pharmaco-engineering systems approach to the development of the next generation of delivery systems with programmable, multifunctional capability. The nature of the PRINT technology takes drug delivery for the first time into the uncharted realm of engineered drug therapies given its unique approach and versatility. PRINT allows for the precise control over particle size, shape, composition, cargo, modulus, and surface properties. PRINT opens up the possibility for key therapeutic parameters such as bioavailability, biodistribution, target-specific cell penetration and controlled cargo release to be simultaneously designed into a therapy.

\section{Cellular Uptake Mechanisms of Nontargeted Organic PRINT Particles}

By taking advantage of the versatility of the PRINT process, we are able to vary and control the amount of surface charge, the size, and the shape of "calibration quality" particles to be internalized to determine the impact these parameters have on the mechanism of cellular internalization. ${ }^{16}$ Several studies have addressed the role of shape and size on cellular internalization. ${ }^{17-19}$ Using commercial polystyrene microspheres and murine B16F10 melanoma cells, Rejman and co-workers have shown that spheres with a diameter less than $200 \mathrm{~nm}$ utilized a clathrin-mediated mechanism for cellular internalization whereas particles greater than $500 \mathrm{~nm}$ were endocytosed using a caveolae-mediated mechanism. ${ }^{18}$ No internalization was seen with particles greater than $1 \mu \mathrm{m}$. Furthermore, surface charge was shown to affect particle uptake with net cationically charged particles being internalized by clathrin-coated pits while anionic particles were endocytosed via caveolae when added to HeLa cells. ${ }^{20}$ Thus size and charge play critical roles in determining which endocytic pathway is used for particle internalization. To our knowledge, few studies have been done on the intracellular internalization of shape-specific organic nanoparticles.

Given this, a series of PRINT particles were fabricated to characterize the cellular internalization mechanisms of non-targeted organic nanoparticles as a function of size, shape, composition, and surface charge (cationic, anionic) in human cervical carcinoma epithelial (HeLa) cells. We have seen that particle shape greatly affects cellular internalization. Particles with a diameter of $200 \mathrm{~nm}$ and a height of $200 \mathrm{~nm}(200 \mathrm{~nm} \times 200 \mathrm{~nm})$ were taken up at a slower rate $(1.2 \%$ of the cell population/minute) than their nonsymmetrical rod-like $150 \mathrm{~nm} \times 450 \mathrm{~nm}$ counterparts (5.2\% of the cell population/minute). We found a strong dependence on surface charge, where after $1 \mathrm{~h}$ of incubation with the HeLa cells, positively charged particles were internalized by $84 \%$ of cells and negatively charged particles were not internalized to any significant amount $(<5 \%)$. We showed that all particles tested possessed minimal toxicity, even at long incubation times (72 h). Transmission electron microscopy (TEM) clearly showed clathrin-mediated endocytosis as one of many methods of endocytosis for these particles.

The most commonly accepted belief for cellular internalization in the literature suggests that particles larger than $200 \mathrm{~nm}$ will not be internalized by nonphagocytic cells; however, we found using a range of techniques that cylindrical PRINT particles as large as $1 \mu \mathrm{m}$ were internalized into HeLa cells. ${ }^{21-24}$ To our knowledge this is the first report of the intracellular uptake and transport of biologically relevant nanoparticles greater than $200 \mathrm{~nm}$ into nonphagocytic mammalian cells. In addition, we find that rod-like, high aspect ratio nanoparticles can be internalized into nonphagocytic cells much more rapidly and efficiently than would be expected based on size considerations alone, suggesting a special role associated with the shape of the particles. We believe the interplay between particle shape and size at constant surface chemistry will undoubtedly play a role in particle targeting strategies and may even shed some light on the rationale behind bacterial pathogen sizes and shapes. 


\section{Preliminary Biodistribution Studies of PRINT Particles}

Preliminary in vivo studies of PRINT particles have now been conducted. Monodisperse 200 $\mathrm{nm} \times 200 \mathrm{~nm}$ PEG-based PRINT particles (78\% (w/w) PEG triacrylate, 20\% (w/w) PEG monomethyl ether monomethacrylate, $1 \%(\mathrm{w} / \mathrm{w}) 2$,2-diethoxyacetophenone, and $1 \%(\mathrm{w} / \mathrm{w})$ para-hydroxystyrene) were fabricated and characterized via scanning electron microscopy and dynamic light scattering. ${ }^{25}$ Incubation with HeLa cells showed no cytotoxicity, even at high particle concentrations. The biodistribution and pharmacokinetics of ${ }^{125} \mathrm{I}$-labeled particles were studied following intravenous (iv) administration in nontumor bearing C57BL/6J mice. The $200 \mathrm{~nm} \times 200 \mathrm{~nm}$ PRINT particles were distributed mainly in clearance organs (i.e., liver and spleen) throughout the time course of the study (Figure 3). The degree of particle accumulation reported in the kidneys, heart, and lungs may over-represent the actual accumulation since the mice were not perfused to clear these organs of blood. The decline in PRINT particle concentration in serum as a function of time followed a biexponential decay, showing characteristic distribution (half-life $=17 \mathrm{~min}$ ) and elimination phases (half-life $=200$ $\min$ ). The rapid accumulation of the particles in the liver and the spleen, as well as the relatively short elimination half-life, indicates that after iv administration the particles were quickly recognized as foreign and removed from systemic circulation by the reticuloendethelial system (RES). This is not surprising considering that the steric coat on the PRINT particles was only a 1000 Da poly(ethylene glycol) (PEG) monomethylether. It has been suggested that the optimal coating for the creation of long-circulating liposomes is $2-5$ kDa PEG. ${ }^{26,27}$ The shorter PEG chains used in the current particle formulation may not offer a radius of protection that is sufficient to effectively block the adsorption of opsonic proteins. Once opsonization occurs, the particles are destined to be cleared by Kupffer cells in the liver and splenic macrophages.

\section{Imaging Modalities for a Dynamic View of Biodistribution}

We have successfully designed PRINT particles that can be conjugated to ${ }^{64} \mathrm{Cu}$, a long-lived positron emitter useful for micro-PET/CT imaging. This work, in collaboration with the Stanford Center for Cancer Nanotechnology Excellence Focused on Therapy Response and the CalTech/UCLA Institute for Systems Biology Nanosystems Biology Cancer Center, allows us to monitor the biodistribution of our PRINT particles in vivo in real time (Figure 4). In order to conduct PET imaging on our PRINT particles, we designed particles with amine handles for the covalent conjugation of both 1,4,7,10-tetraazacyclododecane-1,4,7,10-tetraacetic acid (DOTA), a macrocyclic ligand for the complexation of ${ }^{64} \mathrm{Cu}$, and PEG $5000 \mathrm{~g} / \mathrm{mol}$ ligands. These experiments were performed using a PEG ligand density of $8.4 \times 10^{5}$ PEG chains/200 $\mathrm{nm}$ particle. The PET images suggest a relatively short half-life with most of the particles sequestered in the liver. Currently the group is working on increasing the density of PEG ligands on the surface of the PRINT particles as well as the incorporation of MR contrast agents as a cargo within PRINT particles to complement the PET/CT results described above.

\section{Materials Science Applications}

The generation of high fidelity particles and arrays of nanoscale features afforded by PFPE molding materials opens up a host of key opportunities in the material science sector. Below we discuss the use of PFPE molding and PRINT for applications in optical films and lens arrays, the replication of naturally occurring objects, porous films, and 2D array patterning of inorganic oxides.

\section{Molding of High Performance Optical Films and Lens Arrays}

Imprint lithography is a common tool used to fabricate micro- and nanopatterned embossed films or isolated features for use in photonic and optoelectronic devices. $2,3,28-30$ These patterned films require microscale feature sizes with subpatterns of nanoscale precision for 
surfaces, edges, corners, spacing, and angles for effective light manipulation. Imprint lithography has the potential as a cost-effective alternative to photolithography for manufacturing highly precise optical components and other patterned films and membranes with submicrometer features. ${ }^{31,32}$ However, PRINT is different from traditional imprint lithography since no pressure is applied. The utilization of the PRINT process with a wide variety of optical materials has been demonstrated in batch scale using UV-curable optical resins, conducting polymers, and inorganic oxides. ${ }^{33}$

Figure 5 shows the fabrication of $70 \mathrm{~nm} \times 70 \mathrm{~nm}$ cylindrical features patterned on a glass substrate out of a material whose index of refraction is equivalent to that of the substrate along with the transmission of s-polarized light through the sample. The nanostructured sample shows an increase in transmission for the entire visible spectrum relative to a flat film of the same material. In addition to patterning monoclonal features across a substrate, PRINT is also capable of fabricating features of different size, shape, aspect ratio, and packing density in a single step. Figure 6A is an optical picture of an 8-inch diameter film with five different patterns prepared in a single-step via PRINT. Figure 6B-F shows high-resolution SEM images of the five different areas, depicting the micro- and nanoscale precision of the PRINT process throughout a large area.

\section{Fabrication of Ultra-monodisperse Micro- and Nanoporous Thin Films}

Nanoporous and mesoporous polymeric films are used in a variety of applications such as separations, filtrations, sensors, photonics, optoelectronics, bioactive surfaces, waveguides, and absorbants. ${ }^{34-37}$ PRINT's "top-down" approach allows for the fabrication of micro- and nanoporous membranes with nanometer precision of pore size and shape with the concomitant effect of being able to engineer membranes using a wide variety of high-performance polymers. Traditional "bottom-up" techniques used to fabricate nanoporous membranes such as cast membrane phase inversion, 38 solvent extraction, 39 and block copolymer phase separation ${ }^{40,41}$ have resulted in polydisperse pores or little flexibility in the membrane material. Figure 7 depicts SEM images of microporous membranes fabricated out of PDMS (Sylgard 184), PEG-diacrylate, and the high-performance polymer poly(arylene ether sulfone), showcasing the size and shape control along with the chemical variability of PRINT as a membrane fabrication process. ${ }^{42}$

\section{Replication of Naturally Occurring Objects}

The low surface energy, minimally adhesive, low-viscosity, ambient-temperature photocurable PFPE elastomers enable the replication of naturally occurring objects. ${ }^{9}$ Here, the PFPE liquid precursor is able to be cast over a naturally occurring motif and cured to form an elastomeric mold with the details of the master morphology embedded in the fluoropolymer matrix. The PFPE molding material enables the molding of isolated, weakly adhering, and, in some cases, metastable "soft" nanoscale objects. The PFPE mold can then be used to make replicate objects of the naturally occurring masters in a variety of materials with high fidelity. The molding process has been used to replicate carbon nanotubes, block-copolymer micelles, and virus particles (Figures 8 and 9).

Figure 8 depicts the replication of self-assembled spherical micelles, cylindrical micelles, and metastable toroidal morphologies. Since the micelles are lying on the mica substrate, only one side of the micellar structure is available for molding and replication. The micelles are particularly fragile structures, because they are only held together by noncovalent forces and are freely lying on the substrate. The toroidal morphology shown in Figure 8H-J, formed during the transition from cylindrical micelles to vesicles, ${ }^{43}$ is a metastable morphology that was able to be captured in a PFPE mold and replicated into more stable materials. These structures, for which the "hole" in the toroid has a diameter of less than $20 \mathrm{~nm}$, could potentially be used as 
masks for etching techniques to generate nanoscale features in another material with interesting optical, electronic, or magnetic properties. ${ }^{44}$

The ability to replicate biological structures may provide crucial insight into the importance of shape in biology and lead to advantageous new platforms for imaging and immunotherapies, particularly if chemical cues can simultaneously be incorporated with these biologically derived structure. Figure 9 shows the molding and replication of dispersed adenoviruses on a silicon surface. We were successfully able to replicate the viruses without degradation of the original adenovirus particles, thus further demonstrating the chemical inertness of the PFPE molding process.

\section{Submicrometer Patterning of Inorganic Oxides}

We explicitly use the low surface energy and high gas permeability of cross-linked PFPE-based materials to demonstrate the chemical inertness of fluoroelastomers and have illustrated the use of aggressive chemistries during the embossing and PRINT processes that enable the formation of uniquely patterned, sub-500 nm size, inorganic oxide materials with potential utility in electronic and optical devices. ${ }^{45-48} \mathrm{~A}$ range of oxides including $\mathrm{SnO}_{2}, \mathrm{ZnO}$, ITO (Sn-doped indium oxide), and $\mathrm{BaTiO}_{3}$ have been formed into arrays with precise control over size, shape, and composition (see Figure 10). Figure 11 shows high aspect ratio $\mathrm{TiO}_{2}$ features derived from a sol-gel solution patterned from a $200 \mathrm{~nm} \times 600 \mathrm{~nm}$ PFPE mold on an ITO coated glass substrate. The patterns are able to be formed on both insulating (glass) and conducting substrates (ITO and FTO coated glass slides). It should be noted that when structures are patterned through a sol-gel method, volume loss relative to the original template occurs; however the shrinkage is able to be controlled by varying the initial sol-gel chemistry and annealing rates. There is much interest in nanopatterns of titania for use in titania-polymer solar cells, where a high degree of order and reproducibility is needed to form the desired ideal bulk heterojunction. 49,50

\section{Summary}

In this Account, we have introduced the concept of PRINT, a powerful new particle molding, pattern generation, and replication technique that takes advantage of the unique properties of PFPE elastomeric molds. The low surface energy perfluoropolyether network allows for the production of monodisperse, shape-specific micro- and nanoparticles from an extensive array of precursors and the generation of high-fidelity patterns for a wide variety of materials applications.

\section{Acknowledgements}

This work was carried out by a group of outstanding students and postdoctoral fellows. We would like to thank our co-workers who have made significant contributions to the work described in this Account, including Charlene M. Ross, Moo J. Cho, Joseph L. Templeton, and Rudolf Juliano. The authors would like to acknowledge the collaboration between the Carolina Center of Cancer Nanotechnology Excellence, the Stanford Center for Cancer Nanotechnology Excellence Focused on Therapy Response, and the CalTech/UCLA Institute for Systems Biology Nanosystems Biology Cancer Center, specifically Hsian-Rong Tseng, Anna Wu, and Michael Phelps for their hard work towards the PET/CT project. This work was supported in part by the STC Program of the NSF (Grant CHE-9876674), NIH Grant P01-GM59299, NIH Grant U54-CA119343 (the Carolina Center of Cancer Nanotechnology Excellence), NIH Grant U54-CA119367 (Stanford Center for Cancer Nanotechnology Excellence - Therapy Response), NIH Grant U54CA1 19347 (CalTech/UCLA Institute for Systems Biology Center Nanosystems Biology Cancer Center), and the William R. Kenan Professorship of the University of North Carolina at Chapel Hill and through a sponsored research agreement with Liquidia Technologies. 


\section{References}

1. Xia YN, Rogers JA, Paul KE, Whitesides GM. Unconventional methods for fabricating and patterning nanostructures. Chem Rev 1999;99(7):1823-1848. [PubMed: 11849012]

2. Chou SY, Krauss PR, Renstrom PJ. Imprint lithography with 25-nanometer resolution. Science 1996;272(5258):85-87.

3. Gates BD, Xu QB, Stewart M, Ryan D, Willson CG, Whitesides GM. New approaches to nanofabrication: Molding, printing, and other techniques. Chem Rev 2005;105(4):1171-1196. [PubMed: 15826012]

4. Xia YN, Whitesides GM. Soft lithography. Angew Chem, Int Ed 1998;37(5):551-575.

5. Whitesides GM, Ostuni E, Takayama S, Jiang XY, Ingber DE. Soft lithography in biology and biochemistry. Annu Rev Biomed Eng 2001;3:335-373. [PubMed: 11447067]

6. Lee JN, Park C, Whitesides GM. Solvent compatibility of poly(dimethylsiloxane)-based microfluidic devices. Anal Chem 2003;75(23):6544-6554. [PubMed: 14640726]

7. Rolland JP, Hagberg EC, Denison GM, Carter KR, DeSimone JM. High-resolution soft lithography: Enabling materials for nanotechnologies. Angew Chem, Int Ed 2004;43(43):5796-5799.

8. Rolland JP, Van Dam RM, Schorzman DA, Quake SR, DeSimone JM. Solvent-Resistant Photocurable "Liquid Teflon" for Microfluidic Device Fabrication. J Am Chem Soc 2004;126:2322-2323. [PubMed: 14982433]

9. Maynor BW, Larue I, Hu Z, Rolland JP, Pandya A, Fu Q, Liu J, Spontak RJ, Sheiko SS, Samulski RJ, Samulski ET, DeSimone JM. Supramolecular nanomimetics: Replication of micelles, viruses, and other naturally occurring nanoscale objects. Small 2007;3(5):845-849. [PubMed: 17393549]

10. Euliss LE, DuPont JA, Gratton S, DeSimone J. Imparting size, shape, and composition control of materials for nanomedicine. Chem Soc Rev 2006;35(11):1095-1104. [PubMed: 17057838]

11. Rolland JP, Maynor BW, Euliss LE, Exner AE, Denison GM, DeSimone JM. Direct fabrication and harvesting of monodisperse, shape-specific nanobiomaterials. J Am Chem Soc 2005;127(28):1009610100. [PubMed: 16011375]

12. Xia YN, Whitesides GM. Soft lithography. Annu Rev Mater Sci 1998;28:153-184.

13. Barenholz Y. Liposome application: Problems and prospects. Curr Opin Colloid Interface Sci 2001;6 (1):66-77.

14. Duncan R. The dawning era of polymer therapeutics. Nat Rev Drug Discovery 2003;2(5):347-360.

15. Chien S. Red-cell deformability and its relevance to blood-flow. Annu Rev Physiol 1987;49:177192. [PubMed: 3551796]

16. Gratton SEA, Ropp PA, Pohlhaus PD, Luft JC, Madden VJ, Napier ME, DeSimone JM. The effect of particle design on cellular internalization pathways. Proc Natl Acad Sci USA. 2008in press

17. Champion JA, Mitragotri S. Role of target geometry in phagocytosis. Proc Natl Acad Sci USA 2006;103(13):4930-4934. [PubMed: 16549762]

18. Rejman J, Oberle V, Zuhorn IS, Hoekstra D. Size-dependent internalization of particles via the pathways of clathrin- and caveolae-mediated endocytosis. Biochem J 2004;377:159-169. [PubMed: 14505488]

19. Zauner W, Farrow NA, Haines AMR. In vitro uptake of polystyrene microspheres: Effect of particle size, cell line and cell density. J Controlled Release 2001;71(1):39-51.

20. Harush-Frenkel O, Debotton N, Benita S, Altschuler Y. Targeting of nanoparticles to the clathrinmediated endocytic pathway. Biochem Biophys Res Commun 2007;353(1):26-32. [PubMed: 17184736]

21. Gary DJ, Puri N, Won YY. Polymer-based siRNA delivery: Perspectives on the fundamental and phenomenological distinctions from polymer-based DNA delivery. J Controlled Release 2007;121 (1-2):64-73.

22. Oupicky D, Konak C, Ulbrich K, Wolfert MA, Seymour LW. DNA delivery systems based on complexes of DNA with synthetic polycations and their copolymers. J Controlled Release 2000;65 (1-2):149-171.

23. Conner SD, Schmid SL. Regulated portals of entry into the cell. Nature 2003;422(6927):37-44. [PubMed: 12621426] 
24. Oupicky D, Konak C, Ulbrich K, Wolfert MA, Seymour LW. DNA delivery systems based on complexes of DNA with synthetic polycations and their copolymers. J Controlled Release 2000;65 (1-2):149-171.

25. Gratton SEA, Pohlhaus PD, Lee J, Guo J, Cho MJ, DeSimone JM. Nanofabricated particles for engineered drug therapies: A preliminary biodistribution study of PRINT (TM) nanoparticles. J Controlled Release 2007;121(1-2):10-18.

26. Moghimi SM, Hunter AC, Murray JC. Long-circulating and target-specific nanoparticles: Theory to practice. Pharmacol Rev 2001;53(2):283-318. [PubMed: 11356986]

27. Owens DE, Peppas NA. Opsonization, biodistribution, and pharmacokinetics of polymeric nanoparticles. Int J Pharm 2006;307(1):93-102. [PubMed: 16303268]

28. Huang YY, Paloczi GT, Yariv A, Zhang C, Dalton LR. Fabrication and replication of polymer integrated optical devices using electron-beam lithography and soft lithography. J Phys Chem B 2004;108(25):8606-8613.

29. Menard E, Meitl MA, Sun YG, Park JU, Shir DJL, Nam YS, Jeon S, Rogers JA. Micro- and nanopatterning techniques for organic electronic and optoelectronic systems. Chem Rev 2007;107 (4):1117-1160. [PubMed: 17428024]

30. Ziebarth JM, Saafir AK, Fan S, McGehee MD. Extracting light from polymer light-emitting diodes using stamped Bragg gratings. Adv Funct Mater 2004;14(5):451-456.

31. Quake SR, Scherer A. From micro- to nanofabrication with soft materials. Science 2000;290(5496): 1536-1540. [PubMed: 11090344]

32. Xia YN, Kim E, Zhao XM, Rogers JA, Prentiss M, Whitesides GM. Complex optical surfaces formed by replica molding against elastomeric masters. Science 1996;273(5273):347-349. [PubMed: 8662519]

33. Zhou ZRG, Mar D, Meng X, Orr J, Henn R. Low cost manufacturing of patterned films with nanoprecision. Dig Tech Pap Soc Inf Disp Inf Symp 2008;37:534 -536.

34. Boyle TJ, Brinker CJ, Gardner TJ, Sault AG, Hughes RC. Catalytic membrane sensors. A thin film modified H-2 resistive sensor for multi-molecular detection. Comments Inorg Chem 1999;20(4-6): 209-231.

35. Hedrick JL, Miller RD, Hawker CJ, Carter KR, Volksen W, Yoon DY, Trollsas M. Templating nanoporosity in thin-film dielectric insulators. Adv Mater 1998;10(13):1049-1053.

36. Huo QS, Zhao DY, Feng JL, Weston K, Buratto SK, Stucky GD, Schacht S, Schuth F. Room temperature growth of mesoporous silica fibers: A new high-surface-area optical waveguide. Adv Mater 1997;9(12):974-978.

37. Lu YF, Fan HY, Doke N, Loy DA, Assink RA, LaVan DA, Brinker CJ. Evaporation-induced selfassembly of hybrid bridged silsesquioxane film and particulate mesophases with integral organic functionality. J Am Chem Soc 2000;122(22):5258-5261.

38. Echigo Y, Iwaya Y, Saito M, Tomioka I. A novel preparative method of microporous poly(4,4'oxydiphenylenepyromellitimide) membranes. Macromolecules 1995;28(19):6684-6686.

39. Odom DJ, Baker LA, Martin CR. Solvent-extraction and Langmuir-adsorption-based transport in chemically functionalized nanopore membranes. J Phys Chem B 2005;109(44):20887-20894. [PubMed: 16853708]

40. Hedrick JL, Carter K, Sanchez M, DiPietro R, Swanson S, Jayaraman S, McGrath JG. Crosslinked polyimide foams derived from poly(imide-propylene oxide) copolymers. Macromol Chem Phys 1997;198(2):549-559.

41. Hedrick JL, Charlier Y, DiPietro R, Jayaraman S, McGrath JE. High T-g polyimide nanofoams derived from pyromellitic dianhydride and 1,1-bis(4-aminophenyl)-1-phenyl-2,2,2-trifluoroethane. J Polym Sci, Part A: Polym Chem 1996;34(14):2867-2877.

42. Wiles KBWNS, Herlihy KP, Maynor BW, Rolland JP, DeSimone JM. Soft lithography using perfluorinated polyether molds and print technology for fabrication of 3-D arrays on glass substrates. Proc Int Soc Opt Eng 2006:6151-119.

43. LaRue I, Adam M, Pitsikalis M, Hadjichristidis N, Rubinstein M, Sheiko SS. Reversible morphological transitions of polystyrene- $b$-polyisoprene micelles. Macromolecules 2006;39(1): 309-314. 
44. Brands M, Carl A, Dumpich G. Preparation of large area sub-50 nm polymer nanoring arrays. Superlattices Microstruct 2005;37(6):388-393.

45. Xia DY, Li D, Ku ZY, Luo Y, Brueck SRJ. Top-down approaches to the formation of silica nanoparticle patterns. Langmuir 2007;23(10):5377-5385. [PubMed: 17425349]

46. Yang $\mathrm{P}$, Yang M, Zou SL, Xie JY, Yang WT. Positive and negative $\mathrm{TiO}_{2}$ micropatterns on organic polymer substrates. J Am Chem Soc 2007;129(6):1541-1552. [PubMed: 17243675]

47. Ravirajan P, Peiro AM, Nazeeruddin MK, Graetzel M, Bradley DDC, Durrant JR, Nelson J. Hybrid polymer/zinc oxide photovoltaic devices with vertically oriented $\mathrm{ZnO}$ nanorods and an amphiphilic molecular interface layer. J Phys Chem B 2006;110(15):7635-7639. [PubMed: 16610853]

48. Hampton MJ, W SS, Zhou Z, Nunes J, Ko DH, Templeton JL, Samulski ET, DeSimone JM. The patterning of sub-500nm, inorganic oxide structures using crosslinked perfluoropolyethers. Adv Mater 2008;20:2667-2673.

49. Coakley KM, McGehee MD. Conjugated polymer photovoltaic cells. Chem Mater 2004;16(23): 4533-4542.

50. Watkins PK, Walker AB, Verschoor GLB. Dynamical Monte Carlo modelling of organic solar cells: The dependence of internal quantum efficiency on morphology. Nano Lett 2005;5(9):1814-1818. [PubMed: 16159229]

\section{Biographies}

Stephanie E. A. Gratton is pursuing her Ph. D. under the supervision of Dr. Joseph M. DeSimone where she is focused on nanoparticles for therapeutic and imaging applications.

Stuart S. Williams is a Ph.D. candidate in Chemistry at the University of North Carolina at Chapel Hill under the direction of Dr. Joseph DeSimone. His research focuses on nanopatterning and controlling the nanoscale morphology of materials specifically for applications in optics and photovoltaics.

Mary Napier received her B.S. in chemistry from the University of Illinois and her Ph.D. in chemistry from Northwestern University under the direction of Peter Stair and was a postdoctoral associate at Harvard University under the direction of Cynthia Friend. She is currently a senior research associate at the University of North Carolina in Joseph DeSimone's laboratory.

Patrick D. Pohlhaus is currently working at Liquidia Technologies.

Zhilian Zhou is currently working at Liquidia Technologies.

Kenton B. Wiles is currently a Visiting Professor at Winston-Salem State University in the Department of Chemistry.

Benjamin Mayor is currently is a principal scientist at Liquidia Technologies.

Clifton Shen is an adjunct assistant professor at UCLA.

Tove Olafsen is a postdoctoral fellow at the Crump Institute for Molecular Imaging in the Department of Molecular and Medical Pharmacology at UCLA.

Edward T. Samulski is currently the Cary C. Boshamer Professor of chemistry at UNC-CH. Samulski is also a cofounder of Liquidia Technologies and is helping to commercialize the PRINT technology in material science applications. 
Joseph M. DeSimone is currently the Chancellor's Eminent Professor of chemistry at UNC$\mathrm{CH}$ and William R. Kenan Jr. Distinguished Professor of chemical engineering at North Carolina State University. DeSimone recently launched Liquidia Technologies, along with former members of his laboratory, to commercialize these recent breakthroughs from his laboratory for use in micro-and nanofluidics, soft lithography, and nanofabrication of colloidal particles and displays. 


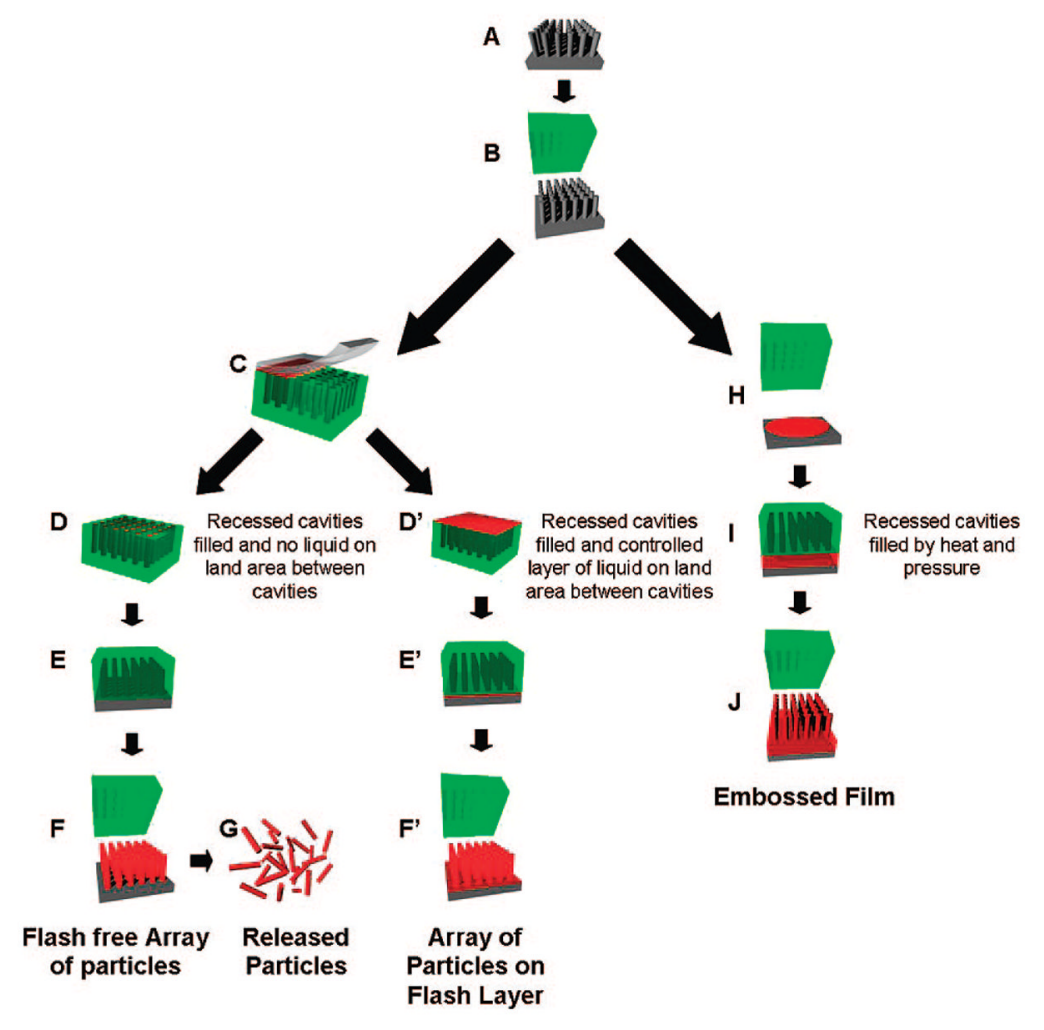

FIGURE 1.

Schematic illustration of the PRINT process and traditional embossing processes: (A) silicon master template; (B) mold release from master template; (C) mold filling via capillary fill with countersheet having a higher surface energy than the PFPE mold; depending on the exact nature of the liquid to be molded and the details of the process, (D) one can fill the cavities only and not wet the land area around the cavities or (D') one can fill the cavities and have a thin layer of liquid on the land area around the cavities. The thickness of the layer of connecting flash layer liquid is determined from the principles associated with free meniscus coating processes with the resulting (E, E') pattern transfer to substrate, $\left(F, F^{\prime}\right)$ mold release from array of isolated features, and $(\mathrm{G})$ dissolution of the harvesting film to yield free particles. As an alternative to PRINT, one can use PFPEs using traditional embossing processes where pressure and heat are applied (H, I) to form an embossed film (J) after the mold is removed. 

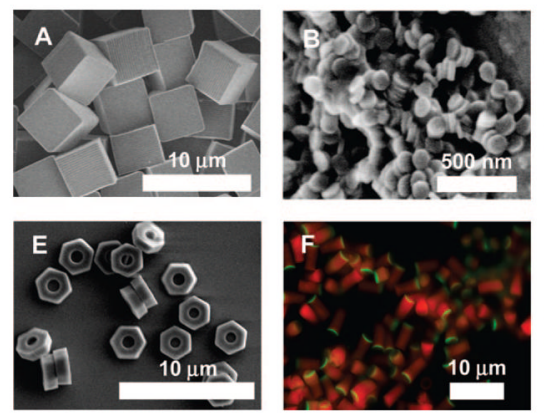
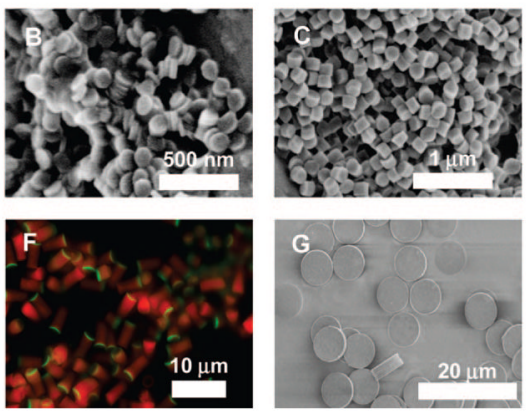

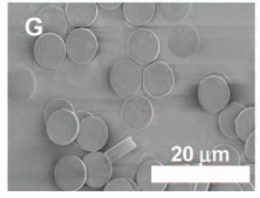

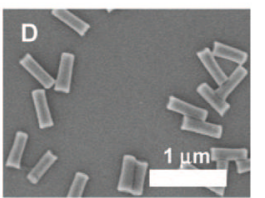

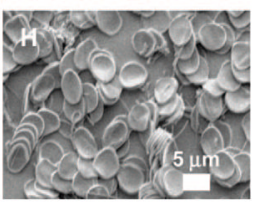

FIGURE 2.

PRINT particles varying in size, shape, surface chemistry, and deformability. The particle composition for all of these particles was approximately the same and included PEG (bulk of the matrix), a cross-linker, and a linker group for conjugation of stabilizing groups (such as PEG) or targeting ligands (such as peptides, antibodies, etc): (A) scanning electron micrograph (SEM) of cube-shaped particles with a cube side length of $5 \mu \mathrm{m}$; (B) SEM of cylindrical nanoparticles having diameter $=110 \mathrm{~nm}$ and height $=35 \mathrm{~nm}$; (C) SEM of cylindrical nanoparticles having diameter $=200 \mathrm{~nm}$ and height $=200 \mathrm{~nm}$; (D) SEM of rod-like PRINT particles having diameter $=100 \mathrm{~nm}$ and height $=300 \mathrm{~nm}$; (E) SEM of $3 \mu \mathrm{m}$ "hex nut" particles; (F) cylindrical PRINT particles containing a covalently attached red fluorophore that have been functionalized on one face with a generic linker group (green fluorophore) that will allow the conjugation of targeting peptides, antibodies, and aptamers region-specifically onto the particle probes; $(\mathrm{G}, \mathrm{H})$ particles for mechanobiology studies having approximately the same dimensions as red blood cells (cylinders with a diameter $=7 \mu \mathrm{m}$ and a height of $1.7 \mu \mathrm{m}$ made from $(\mathrm{G})$ a nondeformable, highly cross-linked hydrogel and $(\mathrm{H})$ lightly cross-linked, deformable hydrogel. 


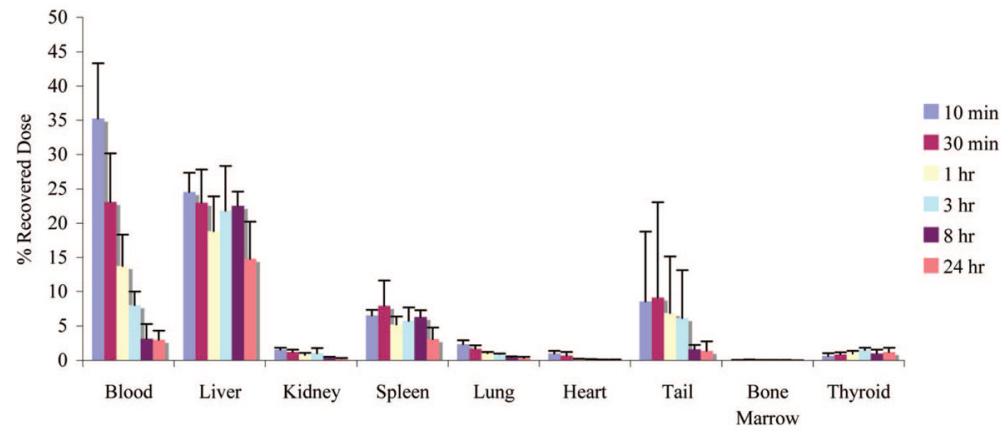

FIGURE 3.

Biodistribution of $200 \mathrm{~nm}\left[{ }^{125} \mathrm{I}\right]-$ labeled PEG 1K PRINT particles in healthy mice. The organ accumulation is expressed as a percent of injected dose. The organ data is presented as the mean \pm SD with $n=4$. Reprinted with permission from ref ${ }^{25}$. Copyright 2007 Elsevier. 


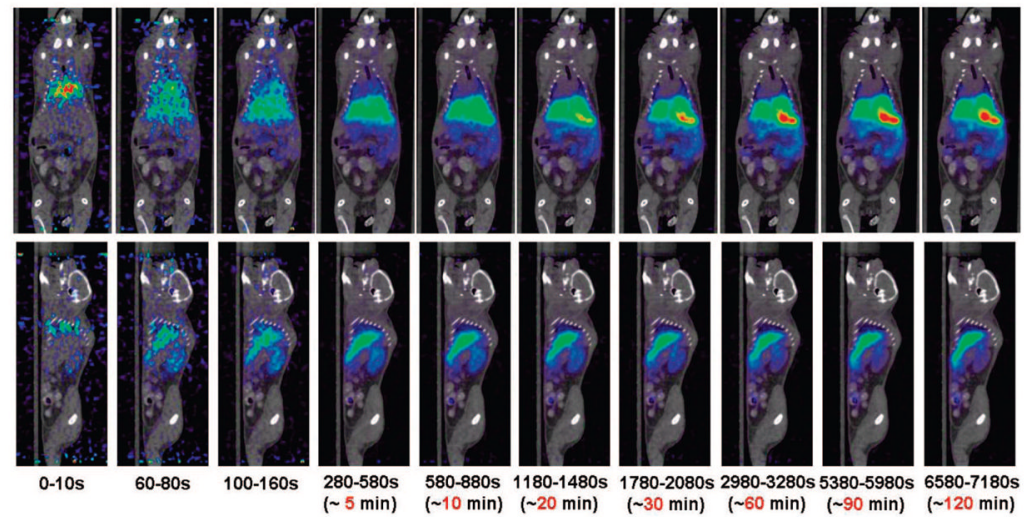

FIGURE 4.

MicroPET imaging with ${ }^{64} \mathrm{Cu}$-DOTA PRINT particles. Time-resolved PET images consist of a $2 \mathrm{~h}$ dynamic scan. The PET/CT images are overlaid. The mouse was injected with 136.2 $\mu \mathrm{Ci}$ of ${ }^{64} \mathrm{Cu}$-labeled DOTA-nanoparticle. Both the coronal view (top), and sagittal view (bottom) are presented. 

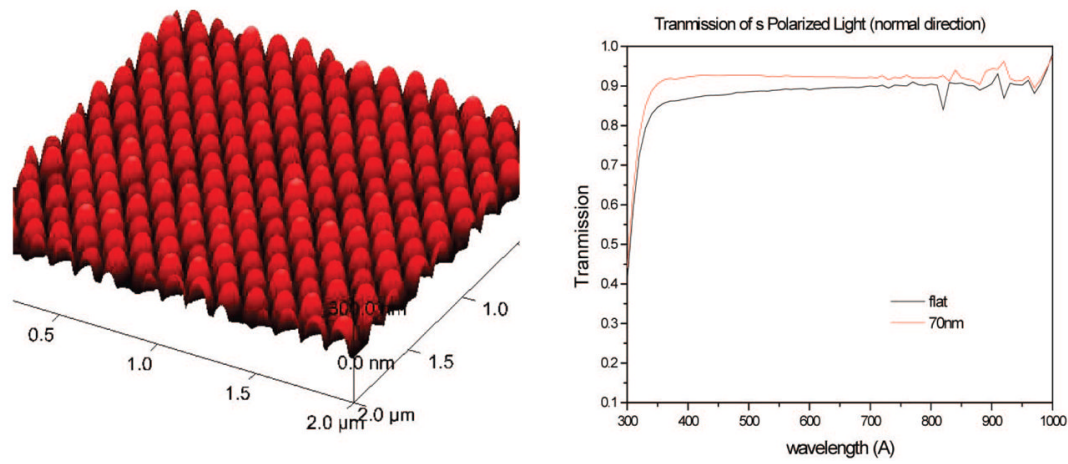

FIGURE 5.

AFM image of $70 \mathrm{~nm}$ features on glass and transmission of s-polarized light. 

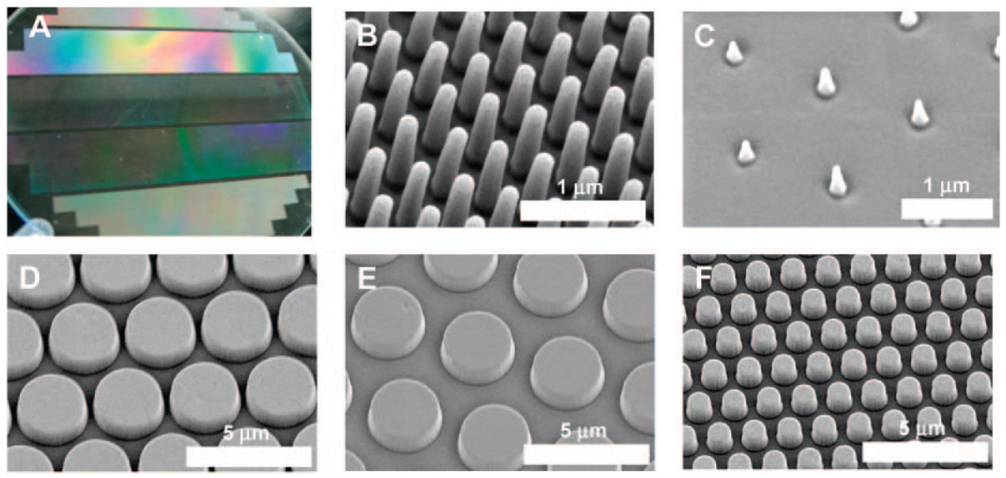

FIGURE 6.

Picture of an 8-in. substrate with (A) five different patterns and (B-F) SEM images of the individual features. 


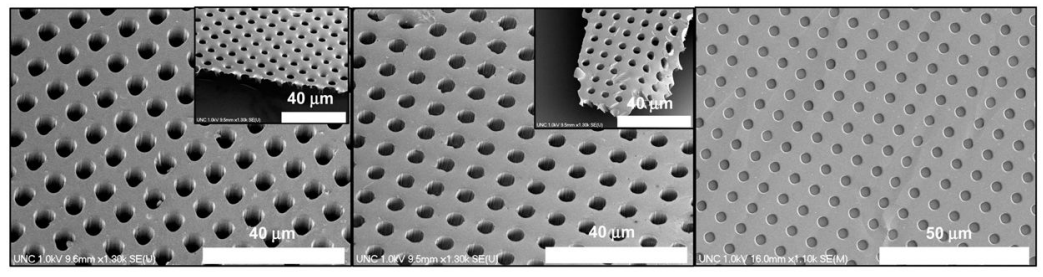

FIGURE 7.

PDMS (Sylgard 184) membranes containing $3 \mu \mathrm{m}$ diameter pores of monodisperse quality (left), PEG-diacrylate cross-linked monodisperse $3 \mu \mathrm{m}$ diameter pore membranes (middle), and poly(arlene ether sulfone) $5 \mu \mathrm{m}$ diameter porous membranes based on biphenol and dichlorodiphenyl sulfone monomers (right). 

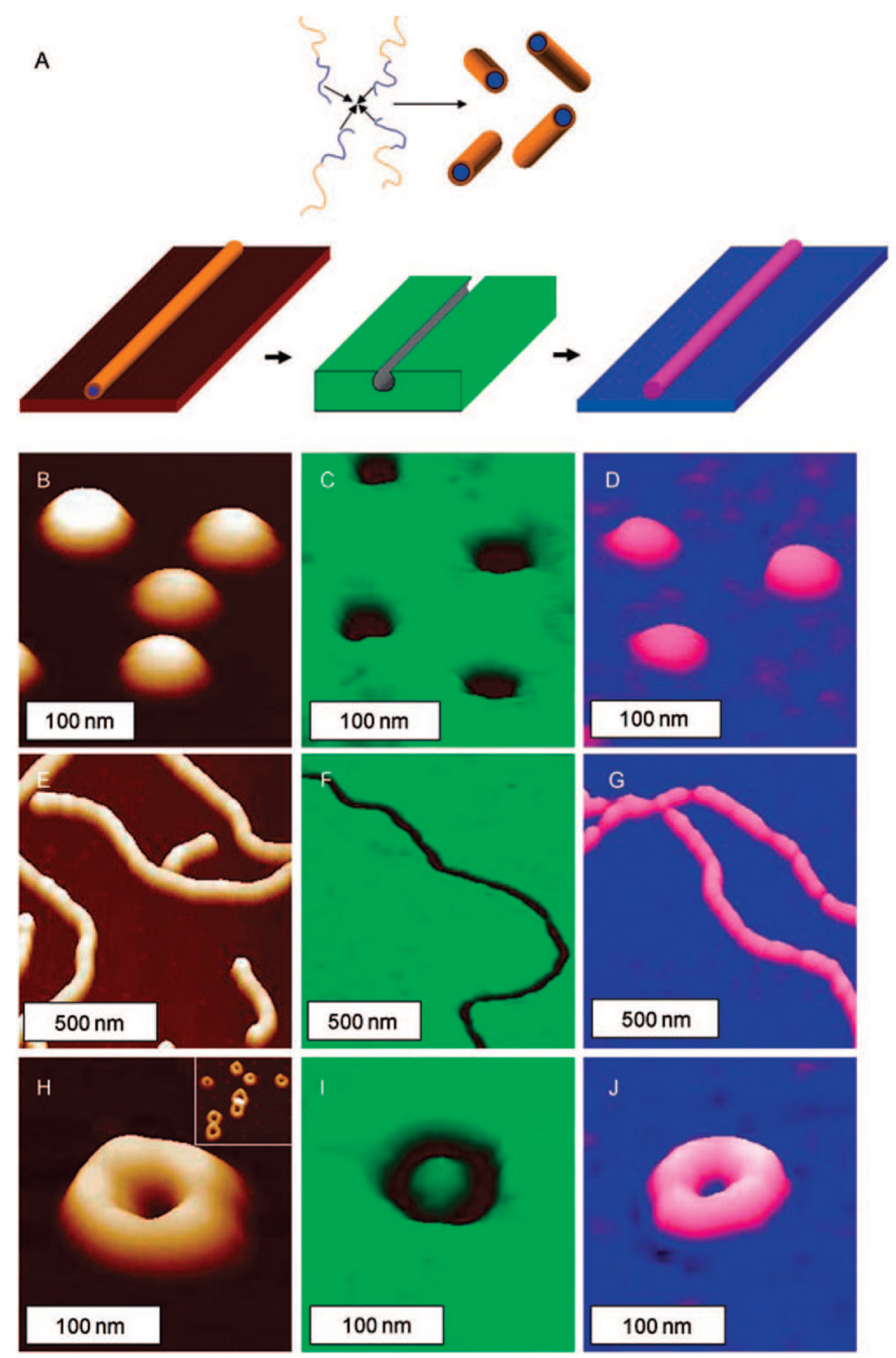

\section{FIGURE 8.}

Polystyrene-block-polyisoprene (PS-b-PI) micelle replication. Depending on the blockcopolymer composition, self-assembly of PS- $b$-PI in heptane results in micelles with welldefined shapes: (A) schematic image depicting self-assembly of micelles and their deposition onto substrates (brown/white), molding (green/black), and replication (blue/magenta); (B) spherical micelle master, prepared by self-assembly of a $39 \mathrm{kDa}-b$ - $94 \mathrm{kDa}$ PS- $b$-PI copolymer and solution deposition onto mica (vertical scale $=100 \mathrm{~nm}$ ); (C) PFPE mold of a spherical micelle master (vertical scale $=20 \mathrm{~nm}$ ); (D) triacrylate replica of spherical micelles (vertical scale $=130 \mathrm{~nm}$ ); (E) cylindrical micelle master, prepared by self-assembly of a $40 \mathrm{kDa}-b-10$ $\mathrm{kDa}$ PS- $b$-PI copolymer and solution deposition onto mica (vertical scale $=300 \mathrm{~nm}$ ); (F) PFPE mold of a cylindrical micelle master (vertical scale $=200 \mathrm{~nm})(\mathrm{G})$ triacrylate replica of cylindrical micelles (vertical scale $=300 \mathrm{~nm}$ ); $(\mathrm{H})$ toroidal micelle master, prepared by selfassembly and deposition of a $21 \mathrm{kDa}-b-4 \mathrm{kDa}$ PS- $b$-PI copolymer and solution deposition onto mica (vertical scale $=45 \mathrm{~nm}$ ); inset, larger AFM image showing a collection of toroidal micelle 
nano-objects (vertical scale $=150 \mathrm{~nm}$ ); (I) PFPE mold of a toroidal micelle (vertical scale $=$ $25 \mathrm{~nm}$ ); (J) triacrylate replica of a toroidal micelle master (vertical scale $=60 \mathrm{~nm}$ ). Reprinted with permission from ref ${ }^{9}$. Copyright 2007 Wiley-VCH. 

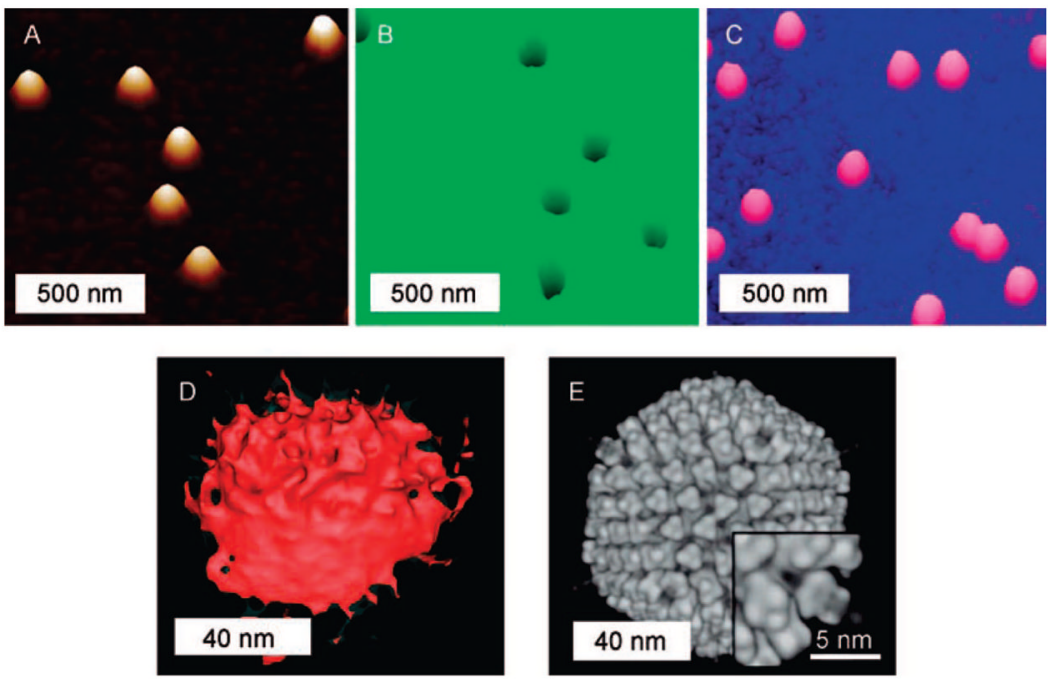

FIGURE 9.

(A) AFM image of an adenovirus master, prepared by depositing adenovirus particles onto a silicon surface (vertical scale $=100 \mathrm{~nm}$ ), (B) AFM image of a PFPE mold formed from an adenovirus master (vertical scale $=50 \mathrm{~nm}$ ), (C) AFM image of a triacrylate/bisphenol A dimethacrylate adenovirus replica (vertical scale $=100 \mathrm{~nm}$ ), (D) transmission electron microtomography (TEMT) reconstruction of a triacrylate/bisphenol A dimethacrylate adenovirus replica, and (E) cryo-electron microscopy reconstruction of adenovirus. Reprinted with permission from ref ${ }^{9}$. Copyright 2007 Wiley-VCH. 


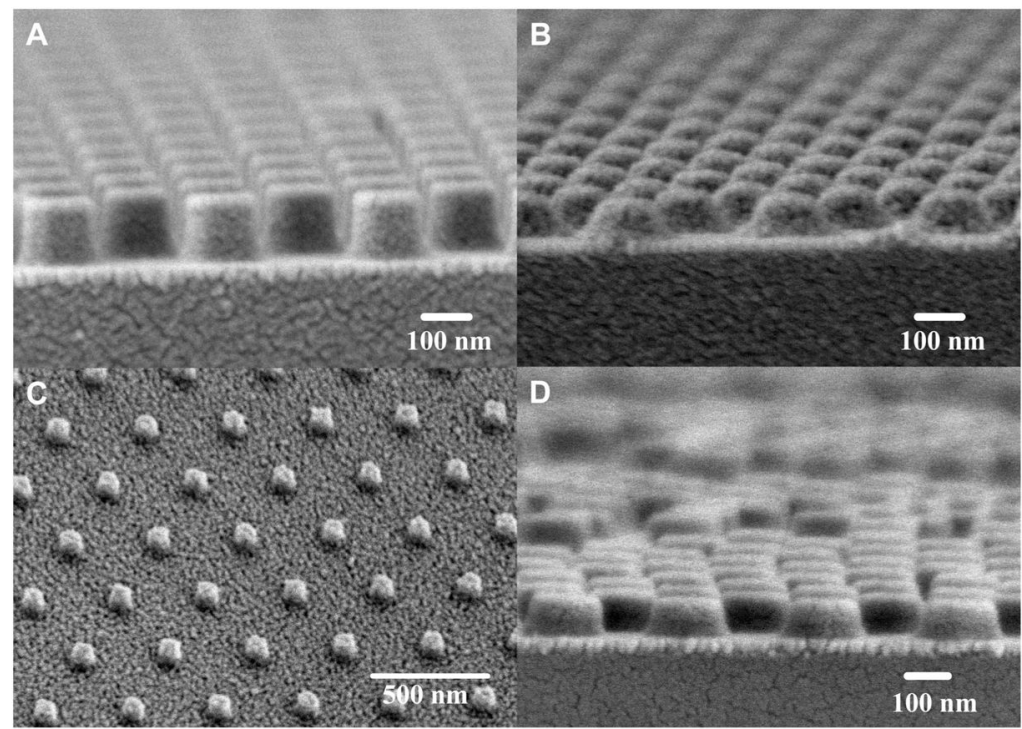

FIGURE 10.

Arrays of features on glass made from a $200 \mathrm{~nm} \times 200 \mathrm{~nm}$ mold in (A) $\mathrm{SnO}_{2}$, (B) $\mathrm{ZnO}$, (C) ITO, and (D) $\mathrm{BaTiO}_{3}$. Reprinted with permission from ref ${ }^{48}$. Copyright 2008 Wiley-VCH. 


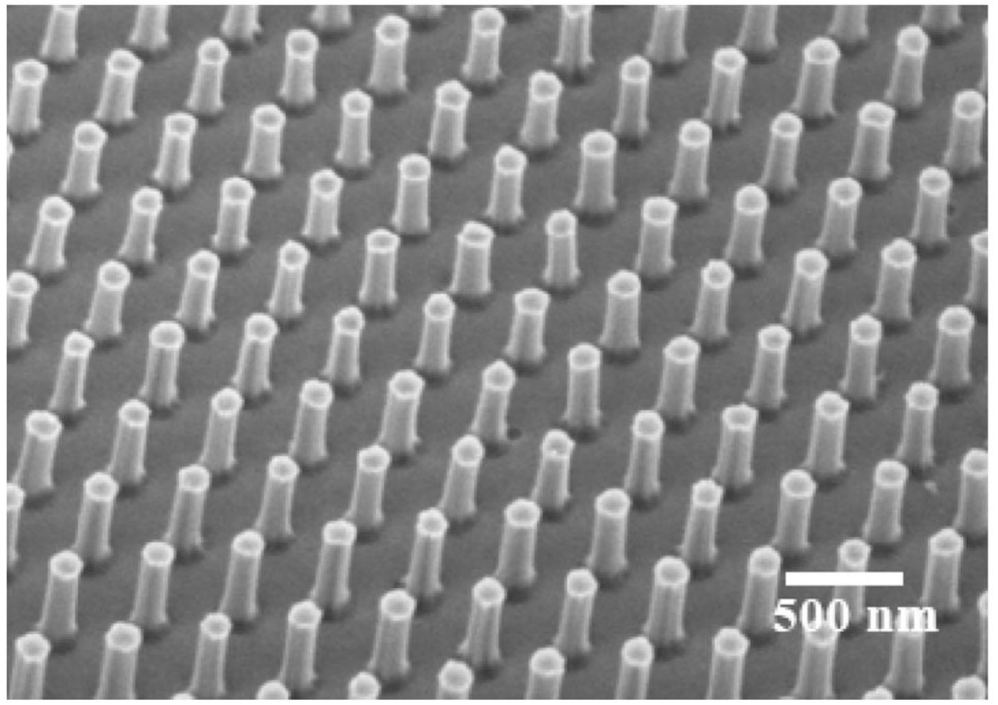

FIGURE 11.

Array of $200 \mathrm{~nm} \times 600 \mathrm{~nm}$ anatase $\mathrm{TiO}_{2}$ features. Reprinted with permission from ref 48 . Copyright 2008 Wiley-VCH. 